\title{
Aplicação de Badges em certificações
}

\author{
Vanessa Nascimento Mendes, \\ Nara Regina Martins Barros, \\ Lucyene Lopes da Silva
}

\section{INTRODUÇÃO}

A globalização e o desenvolvimento das novas tecnologias e a emergência de uma sociedade baseada no conhecimento impõem novas formas de compreender o mundo. Novas maneiras de pensar e agir, inicialmente introduzidas pela Revolução Industrial, sofrem constantes alterações que fazem com que qualificações e competências anteriormente valorizadas se desatualizem. Dessa forma, a exige-se por parte do trabalhador, uma crescente necessidade de estudar e aperfeiçoar-se, servindo assim de alavanca para impulsionar a criação e a oferta de cursos com pequena e média duração. Nesse contexto surgem os badges, emblemas digitais que representam simbolicamente competências e realizações.

Para Ahna, Pelliconea e Butler (2014), os badges têm atraído grande interesse entre os pesquisadores relacionados às áreas de mídia digital e da educação. Randall, Harrison e West (2013) contribuem para essa discussão, distinguindo os conceitos: badges físicos e digitais e os open badges. Segundo esses autores, tanto dentro como fora da escola tradicional, já se utilizam há muito tempo sistemas de reconhecimento físico para atestar: o que uma pessoa conhece (aprendizagem), o que fez (habilidades) ou o que se tornou (papel dentro de uma comunidade). Esse tipo de badge seria simbolizado por emblemas físicos.

Em vez de um emblema físico, os badges digitais, seriam simbolizados por imagens digitais compartilhadas e implementadas para os mesmos fins em comunidades educacionais ou em redes sociais. A Mozilla Foundation baseou-se nesse movimento de badge digital e criou a infraestrutura Open Badges (<http://openbadges.org>) para emissão e gerenciamento de badges digitais com metadados incorporados. Essa infraestrutura é uma plataforma de credenciamento aberta e gratuita que atua como um validador entre emissores e receptores (RANDALL, HARRISON; WEST, 2013).

Para Ahna, Pelliconea e Butler (2014), iniciativas como o projeto Open Badges da Mozilla, expandem e difundem o potencial dos badges no campo da educação aberta, nesse caso, os badges são chamados de open badge. Ainda de acordo com Ahna, Pelliconea e Butler (2014), o Open Badges da Mozilla pode ser considerado um projeto que inclui um número crescente de organizações que planejam 
e emitem badges em diferentes contextos.

Ainda sobre o projeto Open Badges, Randall, Harrison e West (2013) afirmam que ele fornece uma nova maneira de emitir credenciais para que indivíduos atestem conhecimento ou habilidade em um domínio específico. Um open badge inclui metadados que detalham as informações do emissor, os critérios para ganhar o selo e, se desejar, um URL para evidenciar o domínio do ganhador. Esses metadados são enviados com a imagem digital e armazenados nos servidores do emissor. Dessa forma, a estrutura Open Badges oferece um nível de segurança e confiabilidade que os badges digitais comuns não possuem (RANDALL, HARRISON; WEST, 2013).

Além de destacar aspectos relacionados à confiabilidade dos dados do open badge, Randall, Harrison e West (2013) os relacionam com os cursos online massivos abertos (MOOCs). De acordo com esses autores, por meio dos MOOCS, a oferta de ensino que ocorre em ambientes informais e não tradicionais vem aumentando muito, e o open badge representa uma alternativa para reconhecer a aprendizagem fora do sistema educacional tradicional (RANDALL, HARRISON; WEST, 2013).

Ao caracterizar o open badge, Jovanovic e Devedzic (2015) descrevem-no como uma tecnologia importante e relativamente nova que facilita o reconhecimento e credenciamento de diferentes habilidades e conquistas de aprendizado. Por meio de um open badge é possível aumentar ainda mais a motivação de aprendizado (JOVANOVIC; DEVEDZIC, 2014). Randall, Harrison e West (2013) também relacionam o papel do open badge a aspectos comportamentais. Segundo esses autores, o open badge fornece uma maneira de reconhecer a aprendizagem de qualquer ambiente e pode ser a chave para incentivar a aprendizagem ao longo da vida através da motivação e autonomia.

Um badge digital ou open badge podem ser aplicados na educação formal e não formal, como por exemplo, em ações de treinamento e capacitação. Em ambos os casos, é comum atestar o nível de conhecimento e/ou habilidades por meio de certificações.

Buscando conhecer o que já existe de produção científica na área, para realização deste artigo, será conduzida uma revisão sistemática com o intuito de levantar pesquisas relacionadas às certificações e aos badges, visando a investigar a aplicação desse recurso nos processos de certificação.

Esta pesquisa justifica-se ainda no sentido de contribuir para o levantamento e divulgação de dados sobre a aplicação dos badges nas certificações, mais especificamente nos processos de educação formal e não formal. 


\section{METODOLOGIA}

A metodologia que será utilizada neste estudo será uma revisão sistemática de literatura. Segundo Brereton et al. (2007), uma revisão sistemática permite ao pesquisador uma avaliação rigorosa e confiável das pesquisas realizadas dentro de um tema específico.

Para Cordeiro et al. (2007), uma revisão sistemática objetiva responde a uma pergunta de pesquisa claramente formulada, utilizando métodos sistemáticos e explícitos para identificar, selecionar e avaliar as pesquisas relevantes, bem como coletar e analisar dados de estudos incluídos na revisão. Nesse tipo de revisão, busca-se alcançar maior qualidade nas buscas e resultados, ou seja, compreender o "estado da arte" do assunto pesquisado.

Segundo Green et al. (2006), uma revisão sistemática é um tipo de revisão literária que emprega métodos detalhados, rigorosos e explícitos. Ainda para esses autores, o que caracteriza uma revisão sistemática é a busca detalhada na literatura baseada em uma questão de pesquisa que, neste caso, será: “ De que forma os badges podem ser aplicados nos processos de certificação? “

\subsection{COLETA DE DADOS}

Com a questão de pesquisa definida, inicia-se a etapa de busca dos estudos, onde definem-se os critérios de inclusão, bem como as bases de dados a serem consultadas.

A realização da etapa da coleta de dados seguiu os critérios de inclusão de publicações que trazem alguma relação entre os termos-chaves: certificação e badges. Dessa forma, foram examinados todo tipo de produção científica publicada entre os anos 2006 e 2017.

A base de dados adotada nesta revisão será a Scopus ${ }^{1}$. A escolha pela Scopus é devido à relevância dessa base no meio acadêmico na área de Ciências Sociais Aplicadas.

Quanto à seleção das palavras-chave, o Quadro 1 apresenta os termos empregados para a busca dos artigos, ou seja, a lista com as palavras-chave incluindo também a língua inglesa, principal língua utilizada em pesquisa internacional.

1 A base Scopus é atualmente uma fonte importante para bibliotecários, cientistas da informação, editores e pesquisadores que desejam recuperar e avaliar literatura científica. É uma das bases disponíveis aos pesquisadores e instituições brasileiras que têm acesso ao Portal Capes. 


\begin{tabular}{|l|l|}
\hline \multicolumn{1}{|c|}{ Português } & \multicolumn{1}{c|}{ Inglês } \\
\hline Badges & Badges \\
\hline Certificação & Certification \\
\hline
\end{tabular}

Quadro 1: Palavras-chave

Fonte: Autoras, 2018.

A estratégia de busca foi organizada de diferentes formas, a fim de atender as especificidades de cada base de dados. Foram aplicados os operadores lógicos OR e AND para realizar a combinação dos termos utilizados na procura das publicações. Com o objetivo de aumentar o grau de relevância dos artigos à pergunta de pesquisa, novas palavras foram adicionadas à busca. O Quadro 2 apresenta os termos buscados e os resultados obtidos.

\begin{tabular}{|l|c|}
\hline \multicolumn{1}{|c|}{ Termos de busca } & SCOPUS \\
\hline (TITLE-ABS-KEY ("certification" OR "certify") AND TITLE-ABS-KEY & 20 \\
("badge" OR "open badge" OR "digital badge") AND TITLE-ABS- & \\
KEY ("learning" OR "training" OR "education")) AND PUBYEAR > & \\
2005 & \\
\hline
\end{tabular}

Quadro 2: Termos de busca

Fonte: Autoras, 2018.

A busca inicial retornou 20 artigos. Na primeira etapa, foi realizada uma rápida revisão nos títulos e resumos em relação aos critérios de inclusão e exclusão adotados nessa revisão. Artigos irrelevantes, duplicados e pagos foram removidos. Dessa etapa resultaram 13 publicações.

Para filtrar os artigos relevantes ao objetivo desta pesquisa, efetuou-se em uma segunda etapa, a leitura dos resumos, classificando-os em duas categorias: (a) relacionado ou (b) não relacionado ao objetivo da pesquisa. Ao final, apenas 11 artigos tinham relação de alguma forma com a questão de pesquisa. O Quadro 3 apresenta a listagem desses artigos organizados em ordem crescente do ano de publicação. 


\begin{tabular}{|c|c|c|}
\hline Títulos & Autor & Ano \\
\hline $\begin{array}{l}\text { Developing an appropriate design of blended } \\
\text { learning with web-enabled self-regulated } \\
\text { learning to enhance students' learning and } \\
\text { thoughts regarding online learning }\end{array}$ & $\begin{array}{l}\text { TSAI, C.-W.; SHEN, P.-D.; } \\
\text { TSAI, M.-C. }\end{array}$ & 2011 \\
\hline $\begin{array}{l}\text { From proprietary to personalized higher } \\
\text { education - How OER takes universities } \\
\text { outside the comfort zone }\end{array}$ & $\begin{array}{l}\text { OSSIANNILSSON, E.; } \\
\text { CREELMAN, A. }\end{array}$ & 2012 \\
\hline Digital badges in education & $\begin{array}{l}\text { GIBSON, D.; } \\
\text { OSTASHEWSKI, N.; } \\
\text { FLINTOFF, K.; GRANT, S.; } \\
\text { KNIGHT, E. }\end{array}$ & 2015 \\
\hline $\begin{array}{l}\text { A Badge of Honour: Recognising sustainable } \\
\text { teaching principles with open }\end{array}$ & LIU, W.; NORTHOVER, M. & 2014 \\
\hline $\begin{array}{l}\text { Digital badging at The Open University: } \\
\text { recognition for informal learning }\end{array}$ & LAW, P.; LAW, A. & 2015 \\
\hline $\begin{array}{l}\text { Using Open Badges to Certify Practicing } \\
\text { Evaluators }\end{array}$ & $\begin{array}{l}\text { DAVIES, R.; RANDALL, D.; } \\
\text { WEST, R. E. }\end{array}$ & 2015 \\
\hline $\begin{array}{l}\text { Using Mozilla badges to Certify XSEDE users } \\
\text { and promote training }\end{array}$ & KAPPES, S.; BETRO, V. C. & 2015 \\
\hline $\begin{array}{l}\text { Badge it! a collaborative learning outcomes } \\
\text { based approach to integrating information } \\
\text { literacy badges within disciplinary curriculum }\end{array}$ & $\begin{array}{l}\text { FORD, E.; IZUMI, B.; } \\
\text { LOTTES, J.; RICHARDSON, } \\
\text { D. }\end{array}$ & 2015 \\
\hline Badging your way to information literacy & $\begin{array}{l}\text { FOSMIRE, M.; VAN EPPS, } \\
\text { A.S.; JOHNSON, N.E. }\end{array}$ & 2015 \\
\hline $\begin{array}{l}\text { Application of principles of performance- } \\
\text { based assessment to corporate certifications }\end{array}$ & FOSHAY, W.R.; HALE, J. & 2017 \\
\hline $\begin{array}{l}\text { Certification of MOOCS. advantages, } \\
\text { challenges and practical experiences | [La } \\
\text { certificación de los MOOC. Ventajas, desafíos } \\
\text { y experiencias prácticas] }\end{array}$ & KOPP, M.; EBNER, M. & 2017 \\
\hline
\end{tabular}

Quadro 3: Artigos analisados na base de dados Scopus Fonte: Autoras, 2018.

De acordo com os resultados da busca inicial, a associação entre Badges e Certificações pode ser considerada de interesse recente na academia, visto que a maioria dos artigos encontrados foram publicados entre 2014 e 2015, evidenciando-se um aumento considerável no ano de 2015, conforme pode ser visualizado na Figura 1. 


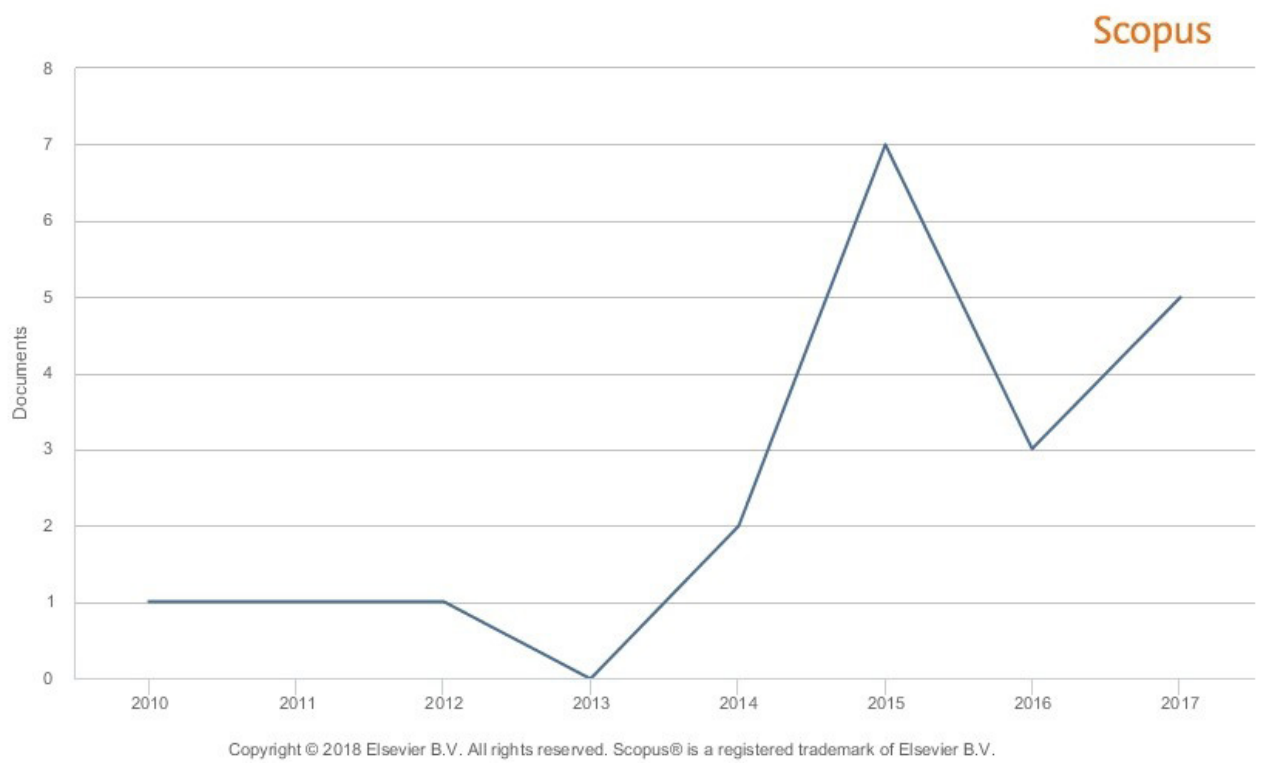

Figura 1: Artigos encontrados Fonte: Autoras, 2018.

Com relação às áreas temáticas, a pesquisa retornou 32 referências, o que pode indicar que os autores atuam em diferentes áreas de pesquisa, isto é, indica publicações multi ou interdisciplinares. As três principais áreas temáticas elencadas na pesquisa, pelo índice de referências retornadas nas buscas, são: Social Sciences (65\%), seguida da Computer Science (50\%) e Engineering (20\%), conforme demonstrada a distribuição do número de publicações. Os dados podem ser visualizados na Figura 2.

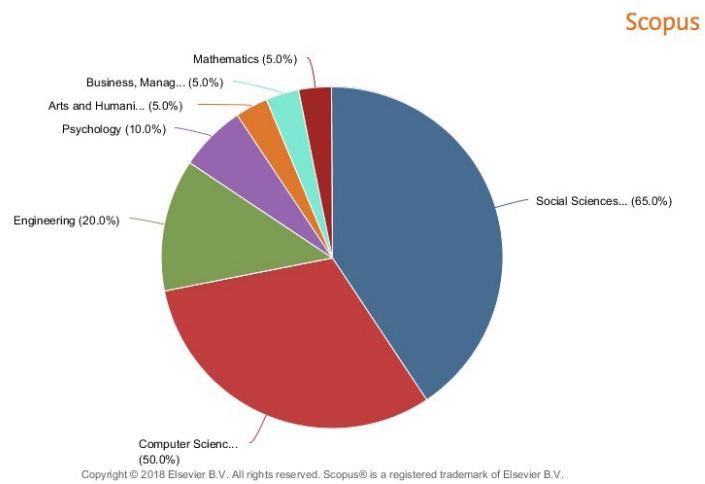

Figura 2: Áreas envolvidas

Fonte: Autoras, 2018. 


\section{ANÁLISE E DISCUSSÃO DOS RESULTADOS}

Quanto aos badges, Ossiannilsson e Creelman (2012) definem que estes são certificados de conclusão cedidos por organizações ou pares que servem para reconhecer habilidades e realizações que tem sido adquiridas de maneira informal e fora dos limites da educação formal. Para esses autores, os badges também servem para reconhecer as competências que são adquiridas informalmente.

Fosmire, Van Epps e Jonhson (2015), ao descreverem o papel dos badges na educação, também associam o seu uso ao reconhecimento de competências. Segundo eles, os badges também chamados de microcredenciamento ou badging, tornaram-se uma maneira popular para certificar a realização de uma variedade de áreas do conhecimento, talvez mais visível na área de tecnologia da informação. Para Fosmire, Van Epps e Jonhson (2015), as instituições de Ensino Superior já começaram a investigar o uso de badges como uma forma de certificar atividades curriculares e extracurriculares, fornecendo uma descrição mais detalhada das competências, habilidades e experiências dos alunos.

Para os mesmos autores, o microcredenciamento fornece uma oportunidade para avaliar o reconhecimento dos resultados de aprendizagem dos estudantes. Law e Law (2015) também associam o uso dos badges no processo ensino-aprendizagem, segundo os autores os badges tem sido utilizados para reconhecer a participação na aprendizagem, bem como motivar os alunos, fornecendo provas de habilidades e realizações no contexto da educação formal e inforrmal. Além disso, Law e Law (2015) apresenta em seus estudos dados atualizados sobre a oferta de cursos em universidades abertas onde a aplicação de badges se caracteriza como certificações alternativas.

Para Law e Law (2015), os alunos veem os badges como um incentivo para participar de cursos online abertos e massivos, os MOOCs. O autor também aborda como os aspectos motivacionais identificados por meio do uso dos badges incentivam o crescimento da gratuidade dos certificados de cursos massivos. Liu e Northover (2014) também trazem os badges para o cenário dos recursos educacionais abertos e afirmam que esse conceito tem sido desenvolvido ao logo dos últimos três anos com grande parte do impulso proveniente do surgimento de MOOCs. Para esses autores, o livre acesso à participação nesse tipo de curso tem crescido, assim, tem-se o desejo de exibir por meio dos badges as qualificações do curso, bem como sua conclusão.

Ainda no contexto dos MOOCs, Kopp e Ebner (2017) realizam um comparativo entre o papel do certificado tradicional emitido no formato PDF e os badges digitais, ambos foram caracterizados pelos autores como uma forma de representar credenciais. 
Segundo Kopp e Ebner (2017), os alunos que se matriculam em um MOOC, por motivos profissionais, desejam obter um certificado tradicional para testar seus resultados de aprendizagem. No dia a dia, usam seus certificados quando procuram emprego ou para atestar o Ensino Superior completo. É reconhecido como um documento oficial, e mesmo que esse documento não passe de um certificado de participação, ele é considerado importante para os alunos de MOOCs. (KOPP; EBNER, 2017).

Ainda de acordo com Kopp e Ebner (2017), a certificação tradicional é baseada fortemente em fatores de motivação extrínseca, o compromisso dos alunos em obter esse tipo de certificado está diretamente relacionado às especificações da qualificação. Se o documento for obrigatório, ele será gerado, caso contrário, o aluno terá um interesse menor.

Quanto ao uso dos badges nos MOOCs, Kopp e Ebner (2017) descrevem que os badges emitidos podem contribuir como um elemento motivador para a conclusão do curso. Para esses autores, os alunos que colecionam suas credenciais na forma de badges têm uma motivação maior ao finalizar um MOOC. Advertem que, embora isso não possa ser medido diretamente, evidenciam em seus estudos que a taxa de evasão daqueles que emitem badges na forma de uma credencial é significativamente baixa quando comparada com a de alunos sem badges.

Para Gibson et al. (2015), os badges, também chamados de emblemas digitais, oferecem novas possibilidades para a educação online. Segundo esses autores, os badges podem ser utilizados como elementos da gamificação, utilizados em combinação com outros elementos, como pontos e leaderboards. Nesse contexto, os badges podem estimular a competitividade entre os alunos, permitindo que eles almejem o alcance de uma meta ou objetivo, e que através de sua conquista adquiram uma reputação. Dessa forma, os badges podem motivar o engajamento contínuo, apoiando os alunos na aquisição de habilidades através de sua performance (GIBSON et al., 2015).

Ainda no contexto da gamificação, Ossiannilsson e Creelman (2012) descrevem os badges como elementos motivadores. Para os autores, o uso de badges na gamificação é uma das principais tendências de tecnologia da educação. Nesse contexto, os badges criam uma sensação de exclusividade e motivam os alunos para níveis mais elevados (OSSIANNILSSON; CREELMAN, 2012).

Tsai, Shen e Tsai (2011) descrevem os badges no cenário da educação profissional. Os autores consideram as certificações profissionais como badges (emblemas) que comprovam habilidades. Nesse contexto, o ensino se concentra em como ajudar os alunos a melhorar suas competências profissionais e passar nos exames de certificação, particularmente em cursos de computação.

Para Ossiannilsson e Creelman (2012), os badges são motivadores comuns 
em serviços de redes sociais como o Foursquare, mas só são válidos no contexto em que são atribuídos. Segundo esses autores, redes sociais como o Linkedin oferecem oportunidades para a avaliação por pares, onde colegas podem escrever recomendações, destacando suas habilidades e realizações. Nesse contexto, os badges poderiam ser exibidos no Linkedin e Facebook, destacando assim aptidões e competências que não são visíveis em certificações formais. Seguindo essa mesma proposta, Gibson et al. (2015) também descrevem que os badges podem ser utilizados em sites de mídias sociais. Assim, os badges sinalizam realizações e têm potencial para se tornar um sistema de credenciamento alternativo, proporcionando o reconhecimento visível em símbolos digitais que apontam diretamente para a validação de avaliações educacionais (GIBSON et al., 2015).

Além de descrever a utilização de badges em redes sociais, Ossiannilsson e Creelman (2012) ressaltam a importância do sistema open badge. Segundo esses autores, $20 \%$ de toda a aprendizagem no local de trabalho é devido às iniciativas de formação formal, os outros $80 \%$ restantes ocorrem de forma natural no processo de trabalho, através da interação com os colegas, avaliações, atividades colaborativas e o autoestudo. Esse aprendizado em grande parte não é reconhecido. A proposta do open badge, que é uma iniciativa do Mozilla Corporation, é justamente o reconhecimento por meio de badges de competências que foram adquiridas informalmente, fora dos limites da educação formal (OSSIANNILSSON; CREELMAN, 2012).

Segundo Liu e Northover (2014), o componente essencial do open badge é a credibilidade do emissor. Para esses autores, é difícil determinar o valor da conquista sem que haja uma evidência ou uma forma transparente de receber o emblema digital. Quanto aos benefícios da aplicação do open badge nos processos educacionais, Fosmire, Van Epps e Jonhson (2015) afirmam que eles sinalizam a realização da aprendizagem, fornecem feedback intrínseco, reconhecem marcos de desenvolvimento e fazem com que os alunos tomem consciência de oportunidades de maior desenvolvimento. Segundo esses autores, a aplicação do open badge pode contribuir para a construção de uma identidade profissional e pessoal.

Conforme já descrito por alguns autores, os badges podem representar uma alternativa para reconhecer a aprendizagem fora do sistema educacional tradicional. No contexto corporativo, Foshay e Hale (2017) afirmam que o modelo baseado em certificados e graus, típico do contexto acadêmico, não atende à gestão de competências necessárias para a força de trabalho. Uma alternativa seria o uso de credenciais. Segundo esses autores, existem diferentes tipos de credenciais, estas podem assumir as seguintes formas: certificações, microcredenciais e certificados. 
As certificações são dadas para pessoas que podem demonstrar capacidade em algum nível ao concluir uma avaliação. As certificações, quando oferecidas por uma agência de credenciamento independente, podem limitar o direito das pessoas ao trabalho que consequentemente estão sujeitas a contestações legais. Geralmente as certificações dependem do rigor da validação por um trabalho ou estudo prático, exigem requisitos de elegibilidade e a especificação de avaliação. A avaliação pode medir conhecimento através de um teste, desempenho em uma condição controlada por meio de uma demonstração ou proficiência. Sua intenção é distinguir pessoas que possuem competência e habilidades daquelas que não possuem. As certificações são temporizadas e expiram exigindo que as pessoas passem por uma reavaliação ou mantenham o nível de competência de alguma forma para que possa manter a credencial. De forma comparativa, os certificados são os menos rigorosos e os mais flexíveis. São concedidos para pessoas que concluem com sucesso um programa de treinamento. Um certificado pode só comprovar a participação, os participantes do treinamento podem ou não ser solicitados a fazer um teste de conhecimento para demonstrar a compreensão do conteúdo do treinamento. Em comparação à certificação, os certificados podem ser facilmente atualizados, refletindo assim as alterações na base de conhecimento e as melhorias na prática. As microcredenciais são um misto de características de certificados e certificações. Seu objetivo é reconhecer a competência em uma área de especialidade, podendo ainda ser utilizada para o reconhecimento de crescimento profissional. Quando oferecidas por uma agência de credenciamento, as microcredenciais têm maior probabilidade de se adequar aos padrões de uma certificação. Quando oferecidas por uma corporação, são utilizadas para avaliações baseadas no desempenho do indivíduo. Possuem maior flexibilidade no escopo, mas normalmente são mais restritas do que uma certificação. Uma microcredencial pode ser validada sobre a competência medida em uma tarefa, um trabalho ou uma função de trabalho. (FOSHAY \& HALE, 2017, p. 3).

Para Foshay \& Hale (2017), os badges não são credenciais, mas sim uma representação visual de uma credencial - geralmente, uma microcredencial. Segundo os autores, um badge pode ser autenticado digitalmente e exibido online em sites e em assinaturas de e-mail, podendo ainda estar no formato físico. Dessa forma, quando comparados aos certificados emoldurados, os badges dão maior visibilidade às credenciais obtidas. 


\section{CONSIDERAÇÕES FINAIS}

Este artigo tem como objetivo demonstrar formas de aplicação dos badges nos processos de educação formal e não formal. Para atingir tal objetivo, buscou-se descrever a aplicação de badges como uma alternativa para certificar os processos de ensino-aprendizagem.

Através da revisão bibliográfica foi possível constatar que os badges são conceitualmente descritos como símbolos ou indicadores de uma realização ou conquista. Reconhecem habilidades ou qualidades do indivíduo. Podem ser simbolizados e compartilhados de forma física ou digital. No contexto da educação aberta, tem-se o open badge. Independentemente da forma como são simbolizados, os badges assumem na educação formal ou não formal seu papel de atestar conhecimentos e/ou habilidades. Podendo ainda ter a função de reconhecer o papel de um indivíduo dentro de uma comunidade. O open badge foi aqui descrito como uma alternativa para reconhecer a aprendizagem não formal, cuja oferta é bastante crescente nos dias atuais.

Por meio da revisão sistemática procurou-se relacionar os termos badges e certificação. De acordo com os artigos analisados, a aplicação de badges em certificações está em ascensão na área educacional. A maioria dos artigos analisados apresentam que essa associação está diretamente relacionada ao reconhecimento de competências e habilidades nos processos de educação formal e não formal.

Os resultados obtidos por este artigo sugerem que os badges, também chamados de microcredenciamento ou badging, representam na atualidade uma maneira de certificar competências, habilidades e experiências em uma vasta área de conhecimentos. A área de Tecnologia da Informação é uma das mais aplicáveis.

Quanto à sua aplicação, os badges podem ser utilizados em diversos contextos, como por exemplo, em processos de certificação em escolas profissionais, universidades abertas e cursos online massivos. Percebe-se ainda que atualmente seu uso é bastante diversificado; várias são as estratégias que combinam o uso de badges com outros recursos educacionais, como por exemplo, as redes sociais, os recursos educacionais abertos e os projetos de gamificação.

Essa diversidade de aplicação pode ser justificada por conta do caráter motivacional dado aos badges. Constatou-se que eles, quando aplicados no contexto educacional, podem apoiar e incentivar a aprendizagem através da motivação e autonomia.

É também no contexto educacional que a gamificação se beneficia quanto ao uso dos badges. Vale aqui mencionar que independentemente do contexto, a gamificação é uma estratégia que objetiva a motivação e o engajamento de pessoas, desse modo, os badges podem ser caracterizados como sendo um elemento motivador na gamificação, proporcionando, assim, um maior nível de envolvimento 
dos indivíduos nas atividades propostas pela gamificação.

Ao considerarmos o cenário atual, caracterizado pela globalização, o mercado competitivo e as constantes transformações tecnológicas, o profissional atual precisa estar em constante qualificação. E a educação formal nem sempre consegue acompanhar essas transformações. Desta forma, a aplicação de badges em microcertificações representa uma alternativa para validar competências e habilidades específicas. A educação formal não consegue atender esse tipo de demanda oriunda muitas vezes do mercado. Percebe-se atualmente uma crescente oferta de cursos online, cujo os temas estão relacionados à educação profissional e corporativa. É também crescente a oferta de cursos online aberto e massivos, do inglês massive open online course (MOOC).

No caso dos MOOCs, os badges podem ser aplicados como um fator de motivação para alunos, estimulando a participação e engajamento. Nesse contexto, os badges podem ainda ser considerados como elementos motivacionais dentro de um projeto de gamificação.

No campo profissional ou corporativo, os badges podem contribuir simbolizando aptidões e competências que não são normalmente reconhecidas em certificações da educação formal. No caso das redes sociais, como por exemplo, o Linkedin, os badges podem representar ferramentas importantes para a construção de uma identidade e reputação profissional.

De forma geral, os achados deste artigo evidenciam os benefícios da aplicação dos badges nos vários contextos da educação formal e não formal, pois, quando utilizados nos processos de aprendizagem, podem trazer benefícios para o aprendizado, promovendo a motivação e o engajamento, reconhecendo competências, habilidades e experiências, fortalecendo, dessa forma, a identidade e a reputação profissional do indivíduo.

Considerando a contemporaneidade do tema abordado e o fato de que os badges terão um papel importante no futuro, entende-se que este artigo não tem fator conclusivo, sugere-se que haja continuidade. Assim sendo, o estudo das seguintes perguntas de pesquisa seria útil para futuras análises: como garantir a qualidade das certificações ou microcredenciais que utilizam badges para representar credenciais? Qual é o impacto que a aplicação de badges tem na vida profissional e social do indivíduo?

\section{REFERÊNCIAS}

AHNA, J.; PELLICONEA, A.; BUTLER, B. S. Open badges for education: What are the implications at the intersection of open systems and badging?. Research in Learning Technology. 22. 10.3402/rlt.v22.23563, 2014. Disponível em: http://dx.doi. org/10.3402/rlt.v22.23563. Acesso em: 13 jun. 2016. 
BRERETON, P. et al. Lessons from applying the systematic literature review process within the software engineering domain. Journal of Systems and Software, v. 80, n. 4, p. 571-583, 2007. Disponível em: <https://doi.org/10.1016/j.jss>. Acesso em: 9 jul. 2016.

CORDEIRO, A. M. et al. Revisão sistemática: uma revisão narrativa. Revista do Colégio Brasileiro de Cirurgiões, v. 34, n. 6, p. 428-431, 2007. Disponível em: <https:// doi.org/10.1590/S0100-69912007000600012>. Acesso em: 16 jun. 2016.

DAVIES, R.;RANDALL, D.; WEST, R. E. Using Open Badges to Certify Practicing Evaluators. American Journal of Evaluation, v. 36, n. 2, p. 151-163, 2015. Disponível em: <https://doi.org/10.1177/1098214014565505>. Acesso em: 12 ago. 2016.

FORD, E. et al. Badge it! A collaborative learning outcomes based approach to integrating information literacy badges within disciplinary curriculum. Reference Services Review, v. 43, n. 1, p. 31-44, 2015. Disponível em: <https://doi.org/10.1108/RSR-072014-0026>. Acesso em: 15 ago. 2016.

FOSHAY, W. R.; HALE, J. Application of principles of performance-based assessment to corporate certifications. Techtrends, v. 61, n. 1, p. 71-76, 2017. Disponível em: <https://doi.org/10.1007/s11528-016-0125-5. Acesso em: 12 fev. 2018.

FOSMIRE, M.; VAN EPPS, A. S.; JOHNSON, N. E. Badging your way to information literacy. In: ASEE ANNUAL CONFERENCE AND EXPOSITION. Conference Proceedings. 2015. (ASEE, v. 122 nd).

GIBSON, D. et al. Digital badges in education. Education and Information Technologies, v. 20, n. 2, p. 403-410, 2015. Disponível em: <https://doi.org/10.1007/s10639013-9291-7>. Acesso em: 17 ago. 2017.

GREEN, B. N. et al. Writing narrative literature reviews for peer-reviewed journals: secrets of the trade. Journal of Chiropractic Medicine, v. 5, n. 3, p. 101-117, Fall 2006.

JOVANOVIC, J.; DEVEDZIC, V. Open badges: novel means to motivate, scaffold and recognize learning. Tech Know Learn, v. 20, p. 115-122, 2015. DOI 10.1007/s10758014-9232-6.

KAPPES, S.; BETRO, V. C. Using Mozilla badges to Certify XSEDE users and promote training. In: ACM INTERNATIONAL CONFERENCE. Proceeding Series. July 2015. (v. 2015). Disponível em: <https://doi.org/10.1145/2792745.2792759>. Acesso em: 12 ago. 2017.

KOPP, M.; EBNER, M. La certificación de los Mooc. Ventajas, desafíos y experiencias prácticas. Revista Española de Pedagogía, v. 75, n. 266, p. 83-100, 2017. Disponível em: <https://doi.org/10.22550/REP75-1-2017-05>. Acesso em: 14 fev. 2018.

LAW, P.; LAW, A. Digital badging at The Open University: recognition for informal learning. Open Learning, v. 30, n. 3, p. 221-234, 2015. Disponível em: <https://doi.or g/10.1080/02680513.2015.1104500>. Acesso em: 12 nov. 2017.

LIU, W.; NORTHOVER, M. A Badge of Honour: recognising sustainable teaching principles with open badges. In: ASCILITE 2014 - ANNUAL CONFERENCE OF THE AUSTRALIAN SOCIETY FOR COMPUTERS IN TERTIARY EDUCATION. Pro- 
ceedings. 2014. p. 505-508.

OSSIANNILSSON, E.; CREELMAN, A. From proprietary to personalized higher education - How OER takes universities outside the comfort zone. Journal of E-Learning and Knowledge Society, v. 8, n. 1, p. 9-21, 2012.

RANDALL, D. L.; HARRISON, J. B.; WEST, R. E. Designing Open Badges for a Tech Integration course. TechTrends, v. 57, n. 6, p. 88-95, 2013. Disponível em: <https:// doi.org/10.1007/s11528-013-0706-5>. Acesso em: 12 ago. 2016.

TSAI, C.-W.; SHEN, P.-D., TSAI, M.C. Developing an appropriate design of blended learning with web-enabled self-regulated learning to enhance students' learning and thoughts regarding online learning. Behaviour and Information Technology, v. 30, n. 2, p. 261-271, 2011. Disponível em: <https://doi.org/10.1080/014492 9X.2010.514359>. Acesso em: 15 ago. 2016. 